\title{
LIFESTYLE RISK FACTORS FOR MCD
}

Results from the PATH through Life Study show that smoking, harmful alcohol consumption, high systolic blood pressure, anxiety and depression in middle age increase the risk of developing a mild cognitive disorder (MCD). MCDs are often the first step to more-serious cognitive decline, which has such a high personal, social and economic cost in societies with aging populations. "The risk factors identified are consistent with past research in more-advanced dementia-this is significant because it further demonstrates that these risk factors have a progressive effect on brain health and cognitive abilities," comments lead author Nicolas Cherbuin (Australian National University, Canberra). $\mathrm{He}$ adds that the results also strengthen the usefulness of a clinical diagnosis of mild cognitive impairment $(\mathrm{MCl})$, "which has been so controversial in the past decade."

The researchers assessed a total of 2,082 people aged between 60 and 64 years for $\mathrm{MCl}$ and other MCDs at two time points, 4 years apart. The participants were randomly sampled from the general population. Notably, the participants were relatively young and were diagnosed with cognitive disorders that occur early in dementia progression, so were more likely to have been representative of the broader population and at a less advanced stage of illness than the participants in previous studies. "Most previous research has investigated the risk factors of cognitive decline and dementia in older individuals (70-80 years) who were often sampled from specific populations attending memory clinics or general practitioners' practices," explains Cherbuin.

The results reveal that smoking is deleterious for brain health and cognition, as is harmful and excessive alcohol consumption. Moderate alcohol consumption, however, seems to be protective-more so than abstinence. High systolic blood pressure represents a risk factor for cognitive dysfunction, whereas higher diastolic pressure seems to be protective. Anxiety and depression were also risk factors for developing $\mathrm{MCl}$, but the apolipoprotein $\mathrm{E} \& 4$ genotype, which is the main genetic risk factor for late-onset Alzheimer disease, was not a risk factor in this cohort.

Although the findings confirmed some previous observations, particularly with regard to smoking, there were some surprises. "The nonlinear effect of alcohol consumption with increased risk for abstainers and for heavy drinkers was particularly interesting," says Cherbuin. He also points out that the protective effect of higher diastolic blood pressure was unexpected.

This and other recent research strongly suggest that the pathological processes leading to cognitive decline start early, probably in early adulthood. "From a public health perspective it is critical to tackle the risk and protective behaviors as early as possible to maximize their beneficial effect on cognitive health," stresses Cherbuin. Such an approach entails increasing health literacy and finding out more about social and personality factors that influence the translation of knowledge into behavior. "Early identification of those at higher risk of cognitive decline is crucial. This would enable us to offer them support, develop effective interventions to decrease risky and increase protective behaviors, and to detect those already affected by cognitive impairment and dementia so they can benefit most from the treatments available," he concludes.

\section{Kathryn Senior}

Original article Cherbuin, N. et al. Risk factors of transition from normal cognition to mild cognitive disorder: the PATH through Life Study. Dement. Geriatr. Cogn. Disord. 28, 47-55 (2009). 